\title{
Research on the "Trinity" Construction of College Students' Psychological Crisis Prevention and Intervention
}

\author{
Hengshan Yi \\ Wuhan University of Science and Technology \\ Wuhan, China
}

\begin{abstract}
Nowadays, owing to the influence of social environment, family environment and school environment, college students are faced with academic pressure, employment pressure, economic pressures and interpersonal relationships pressure and so on, which have a serious impact on the healthy growth of college students and lead to frequent psychological crisis events. Colleges and universities should focus on the reasons and contents of college students' psychological crisis, and strive to build the "trinity" protection system for college students' psychological crisis prevention and intervention to protect the healthy growth of college students.
\end{abstract}

Keywords-college student; prevention and intervention of psychological crisis; the Trinity

\section{INTRODUCTION}

With the continuous development of society and enormous change on family and school environment, now college students have to face academic pressure, employment pressure, economic pressure and communication pressure, thus it causes more and more students' psychological problems which greatly impact on their coordinated development of ideological and moral quality, scientific and cultural quality and health quality. Strengthening effective intervention of college students' psychological crisis risk and ensuring the healthy growth of college students has become an important mission of higher education. In practical work, if universities can skillfully operate the psychological crisis intervention mechanism and emphasize the construction of trinity protection system on students' psychological crisis, it will effectively guide the healthy growth of college students.

\section{THE ANALYSIS OF THE RoOT CAUSES OF COLLEGE STUDENT'S PSYCHOLOGICAL CRISIS}

\section{A. The Influence of Social Environment Factors}

Our country is in the period of rapid economic development, and people's ideological concepts, moral concepts and lifestyles have undergone profound changes. All kinds of streams of thoughts, values and views constantly impact on the thinking of students, some of whom are experiencing inappropriate thought and unacceptable behavior such as belief confusion, unclear faith, distorted value orientation, and behavior imbalance to varying degrees.
Meanwhile, with the increasingly fierce social competition, the tense pace of life and the enormous pressure of work make them feel physically and mentally exhausted. The occurrence of some bad competitions and corruption in the society is undoubtedly a challenge to the weak-willed college students, which will inevitably aggravate their psychological burden. When facing obstacles in life, employment, study, interpersonal communication and so on, college students may produce negative psychological reactions, leading to psychological crisis and problems.

\section{B. Influences of Family Environment}

Family plays a vital part in a person's growth. One's cognition often starts from their family environment and the behaviors of family members. Different family education and environment result in totally varied consequences.

1) Difficult school work: Some of the families are strict with kids' education, and these parents tend to pay much attention to their children's growth, and push hard on their studies in fundamental education ,but less support their kids' other hobbies. Orders and censure are often used to force their kids into doing something. After the children being enrolled in colleges, free and independent environment is provided; however, without parents' supervision, these children have decreasing learning awareness, leading to difficulties in studying.

2) Difficulties in interpersonal relationship: Some parents always spoil their children and are afraid that the children are frustrated. The college students growing in such family environment are lack of compassion and self-restraint, and are at a loss when meeting frustrations. Some parents indulge children, which causes the children to be self-centered and willful without family education and respect for others, so they are hard to adjust to group living, especially life in dormitory. In addition, some parents are incompatible or divorced, which emotionally affects children, resulting in psychological reaction like being irritable and constrained.

3) Difficulties in family economics amid the era: The increasing economic development and living standard improvement enable most families afford children's college fees. However, there are still many students in rural areas who 
can go to college need to do part time jobs to make their ends meet during the academic year owing to their financial difficulties. College is a small society where the gap between the rich and the poor is further widened. As the offspring of wealthy families and the officiallings come in college, the huge differences in basic necessities such as their clothes, food, ways of transportation and living environment bring great psychological impact on the students coming from poor family. As a result, they may become diffident and hostile to the rich

\section{The Impact of Campus Environment}

1) Psychological maladjustment caused by the transformation of environment and role: Before going to college, life has parents arranged. While entering the college, one has to be independent, which makes many students very uncomfortable. The contrast and contradiction of dependence and independence causes their fear and confusion to college life. What's more, because of the great changes in their learning and living environment, they can easily feel nervous and psychologically stressed.

2) Pressure of study and employment: Many students are among the best in their secondary school and high school; however, such advantage is no longer obvious when in college. As the competition of study is fierce, part of the students doubt their learning abilities and even abandon themselves due to the issues like unremarkable grades or failing classes. After the increased enrollment of colleges, there are about seven million graduates annually, so the social competition is more and more intense. The government makes the employment issue a significant task to spare no effort to solve. Whether it is "the hardest year of employment "claimed by the media, or the new measure of encouraging 'mass entrepreneurship and innovation' taken by the government, the undergraduates all suffer great psychological burden, and part of them have fear of employment. Some students lose confidence and are pessimist about their prospect after several failures in applying for jobs. The vulnerable even choose to commit suicide and bring irretrievable consequences to family, college and society.

3) Bad relationship: Relationship now has become a much sensitive problem in today's college environment. Quite a lot of students are in a dilemma. Some college students would close their heart, unable to trust others, and lack the experience of interpersonal communication. As a result, they are always under stress and loneliness. Some other students lack the education from family; therefore, they don't know how to respect others and may be capricious or bad-tempered. These factors could make it difficult for them to fit in the dormitory life. In the end, dormitory contradictions happen easily, and do harm to student's physical and mental health. Besides, the maturity of physiology makes them want to approach opposite sex. But because of the immaturity of mind, in the process of association, they are tending to be less serious and much sightless. Under this condition, they easily get in the relationship misunderstanding, and finally enter the condition of unstable emotion, mental conflict, then may behave disorderedly. If they don't get the correct leading, they will possibly commit suicide.

4) Rampancy of unhealthy campus culture: Nowadays, some undesirable phenomena and culture are eroding college, and doing harm to the pure and noble college culture. For instance, many students cost too much energy in aimless superstar worship and get lost in life; some students are keen on surfing Internet and on-line games, being divorced from the real life, and they escape from the real life and are obsessed in the virtual world; some students are addicted to table pokers and wines, or paying too much attention to romance. These bad behaviors easily change one's personality and affect cognition, emotion and mental state, and even cause a split of personality, which badly impair the mental health of a university student.

\section{THE PREVENTION AND INTERVENTION OF COLLEGE STUDENT'S PSYCHOLOGICAL CRISIS SHOULD START FROM MECHANISM}

The work of intervening crisis not only needs the individual effort of instructors but also requires mechanism and management. During the procedure of crisis intervention, the cooperation of all parts is critical. From the point of personnel, the crisis intervening parts usually include related departments and leaders at school, professional staffs, teachers, family members and friends, and the main student cadres. Therefore, it has to build up an effective Internet protection mechanism, which is a linked mechanism of "Trinity" involving related departments and leaders, professional staffs and instructors.

The core of this "Trinity" mechanism is to set up a system to monitor and deliver crisis information which is a proactive managing procedure from top to bottom. First, psychological test should be required as the early monitoring and warning measure of psychological crisis; besides, early alert messages would be sent to relevant departments and personnel through evaluation and forewarning, which can prevent the burst of psychological crisis of college students. This is a kind of preliminary management. During this process, college psychological education departments play an important role. As well, the professionals should find out the student who has psychological crisis in time by psychological counseling and tutorial lectures, and report to the relevant administrative departments and make further intervention. It is management during the process. Moreover, the instructors can monitor the psychological crisis and give feedbacks by communicating with parents, teachers and student leaders, and later make decisions based on the information and intervene actively, so as to control and minimize the negative impact of the incoming psychological crisis. In the process of intervention, it is important that the personnel of each department should work together. The professionals, instructors, classmates and parents are normally the finders of psychological crisis. Only reporting to the relevant departments and school leaders and working together can save precious time and create advantageous conditions for next intervention. 


\section{PREVENTION AND INTERFERENCE OF PSYCHOLOGICAL CRISIS OF COLLEGE STUDENTS SHOULD BE IMPLEMENTED FROM 3 DIMENSIONS}

Mental health education of college students is an all-round systematic program which needs to be realized through the "trinity" education system containing society, family and college. In such a system, society, family and college are integrated and mutually complementing and interworking, and they play roles together. As colleges, we can provide harmonious and relaxed environments for healthy growth and success of college students from 3 dimensions (relative departments of college, counselors and professional staff), and create an excellent development space for them.

\section{A. Relative Departments of College}

1) Establish mental crisis intervention centers for college students to be responsible for the daily routines of their mental crisis research and intervention: After new students enter the college, the psychological counseling center of college will conduct psychological quality general survey for all new students to have a comprehensive understanding of personality characteristics of them and psychological barriers and symptoms in their study and adaptive processes of life, lay emphasis on the students that may have psychological crisis, implement the prevention and transformation works of crisis, and have a relative definite understanding and grasp of students of the whole college from a dynamic perspective; strengthen the construction of professional teams of psychological counseling, irregularly launch academic research and educational trainings, etc., and improve the workers of mental crisis prevention and intervention, especially the technology and level of frontline counselors and teachers to further improve the intervene effects.

2) Make efforts to construct an atmosphere and form the pattern of involvement of all people: Prevention and intervention of college student mental crisis is not only the work of a small number of instructors, the vast teachers and students should all be mobilized to actively take part in it, especially the mobilization of activeness of teachers. They should actively guide the students at the same time when they teach professional knowledge, and conduct education on ideology, politics and mental health for them; meanwhile find problems and timely feedback and report, thus forming a mental health guidance atmosphere and pattern that is "mobilized, taken part in and supported by all people".

3) Promote optimum distribution and integration of regional resources, and give full play to the radiation and driving effects of advantaged colleges: The key point of mental crisis intervention of college students is to timely select proper and professional disposal and coping model during the intervention process and help the students to overcome difficulties. Mental crisis intervention of college students is a professional and systematic program, whose effective implantation relies on a quickly responding expert team and needs further study among colleges to explore the solutions together and realize the resource sharing. Establishment of a mental crisis intervention system should be started from all aspects including prevention mechanism, integration of talents and professional institutions, etc., and then a mental crisis intervention team consisting of psychology experts, researchers of public health and workers of mental health control can be constructed. On one hand, we can make use of expert resources of advantaged colleges to affect the mental crisis intervention of college students at surroundings and thus promoting the effectiveness of realtime intervention of crisis; on the other hand, integration of professional forces can also be beneficial for research of crisis and integration experience, and then better prevent the crisis.

4) Strengthen the construction of campus culture and build a food education environment: During the on-campus study time, campus culture has a vital effect on the students. Relative departments need to closely coordinate with each other, strictly check on, strengthen the campus cultural construction, build an active, health and elegant campus atmosphere, launch cultural and sports activities with high grade, high quality and high participation rate to strengthen the cohesion of student groups, generate collective sense of honor, to please and relax the body and mind of each student participating in the activities and to educate, inspire and purify their minds. Lay emphasis on the optimization and beautification of college environment, strengthen landscape construction of campus, which is silently beneficial for the physical and mental health of students through campus landscape construction with the sense of times and arts. Build excellent school spirits, styles of study and teaching and place students inside it, molding their characters, purifying their minds, forming active psychological qualities and promoting coordinated developments of college students.

\section{B. Ideological and Political Tutor}

1) Increase communications with students and establish interview system: Establish the service theory of "studentoriented", combine the ideological and political education with the psychological counseling, and establish mental health interview system to provide more chances for students to communicate. If the students are not that active, their conditions can be understood from multi aspects including students, class teachers, course teachers and mental counselor, etc. through visiting dormitories and classes. Apply psychological counseling technology during the communication process with students, do not be hurry to deny the students or make decisions for them when faced with problems in their growth including interpersonal relationships, academic supervision and emotional problems, etc. Sincerely discuss with the educated students, accompany them to face the problems, make them clear that not only him or herself is faced with such problems, instead, many students have experienced such confusions, and the key point is how to treat and face them, analyze the advantages and resources that they have, encourage them to actively seek for help, sincerely 
understand their real thoughts through acceptance and hearing, thus making them explore and discover themselves meanwhile seek for and solve problems. When faced with students with the suicidal tendencies, keep calm, patiently hear what they say, accept them and do not make any judgment, let them talk about their own sense and judge whether the students have the tendency or behavior to commit suicides through talk and observation, do not permit the students to keep secret for their thinking of suicide, obtain help from others as far as possible, if the suicide tendency of the students is high, then immediately adopt urgent crisis management measures. Be the guider of thoughts of college students, the helper of their lives, the counselor of their mentality and the schemer of their futures through understanding their mental conditions and providing necessary guidance in their study, life and mentality, combine the solution of problems of thoughts and mentality with solution of practical problems, thus winning the belief of college students and promoting their healthy growth and success.

2) Give play to the function of key role of students, and strengthen the peer counseling: Student party members, party and youth league branches, student union, class leaders and head of dormitories are backbones of the students, they are both the organizer and leader of all kinds of activities and the important link between the counselor and students, they give play to the function of a bridge, have built certain prestige among student groups, and have a strong influence and appeal to the students. Thus, counselors should conduct professional guidance and trainings for the student backbones, making them give full play to the active functions as backbones, make clear their responsibilities and let them undertake the responsibility as peer counselors, make full use of chances including mid-night meetings of dormitories, dinner parties and collective physical exercises, etc. to enable them to communicate with their intimate friends and classmates, to serve for classmates and actively care for them, student backbones can also play the role of bridge for communication, in which they timely reflect serious problems and conditions to teachers and enable professional teachers to conduct mental guidance for them.

3) Permeate the theory of crisis prevention into daily education: It needs to strengthen daily publicity and education of crisis prevention, counselors can make use of patterns including class meetings, collective activities and new media, etc. to strengthen the publicity of mental crisis prevention knowledge, to propagandize the significant meaning of mental health for personal growth and success and difficulties that may come across in growth processes, how to seek for help when coming across difficulties and how to adjust themselves, to enhance the supporting system and the psychological endurance when faced with difficulties and how to identify and help students in need, etc. Strengthen the construction of class cohesion, advocate mutual care, friendship and solidarity among collective members, create a collective environment that is full of understanding, relaxation and belief, form a strong collective cohesion and make students have the sense of belonging, thus they can pay back and be permitted to the class. Strengthen dormitory culture construction. As the site with the most dense group activities of students, dormitories become the important position for colleges to educate students as a second classroom, which is window for students to acquire information and a channel to communicate on ideologies, and the formation and changes of philosophy and value system of students are affected by the dormitory atmosphere to a large extent. Counselors can promote optimum communications through forms of group guidance, etc. to build an active and healthy dormitory culture and atmosphere, to transmit positive energy among students and solve the generation of mental crisis from the root.

4) We should strengthen communication with parents, realize the linkage between home and school, let parents directly participate in and closely cooperate with heart crisis intervention work, and improve the effectiveness of psychological crisis intervention of college students: First of all, students have psychological crisis, which is largely caused by family conflict and education bias. The relationship between parents and children is the most important factor. Secondly, the student's personality comes from the family and the parent is the guardian, is more familiar with the child's character characteristics; Once again, the important link of psychological crisis intervention, such as escort, referral, treatment, etc., needs parental support and cooperation. Therefore, the understanding, support and cooperation of parents are an important guarantee for psychological crisis intervention. As a student working system teacher, counselors should communicate with parents actively and guide them to correctly understand the role of participants and guardians in the psychological crisis intervention of college students. Encourage them to actively participate in the management process of the school, cooperate with the corresponding work, so as to realize the effective linkage and cohesion of the school and the family. On the basis of respecting students' personal freedom and individual independence, in order to promote the development of college students' mental health, we should strengthen the communication and connection with students' families. The two sides jointly pay attention to the students' psychological and behavioral tendencies, analyze the causes of the problems together, find the point of intervention and establish close contact with the school's psychological counseling center. When students face a serious psychological crisis, we should take appropriate intervention measures, timely transfer students to psychological counseling professionals, assist the consultant in the follow-up evaluation, help students survive the crisis and enhance their ability to adapt to the environment.

\section{Professional Staff}

1) Strengthen publicity and guide college students' selfeducation: Professional teachers should make full use of 
classroom teaching, lectures, school radio, TV, Internet, newspaper and publicity columns, and widely publicize and popularize the methods and approaches of mental health consultation. Popularize mental health common sense, strengthen student's autonomous participation consciousness, enhance and improve mental health level consciousness, seek help voluntarily; Educate students to adjust themselves so that students can motivate themselves, improve the ability of selfawareness and self-education to deal with all kinds of stress and psychological crisis properly.

2) Carry out psychological consultation work effectively: Psychological counseling teachers should carry out psychological counseling effectively and effectively. Provide regular and timely mental health education guidance and service to students through individual consultation, group counseling activities, psychological behavior training, letter consultation, hotline consultation, Internet consultation and other forms.

3) Conduct regular mental health seminars and group competitions: Psychological professional teachers can carry out targeted mental health seminars, or organize students to participate in group competition activities to let the students overcome the psychological crisis in practice according to students' academic and employment difficulties, interpersonal difficulties, family financial difficulties and other pressures.

\section{CONCLUSION}

To establish college students' psychological crisis prevention and intervention system is the inevitable requirement of psychological crisis prevention and intervention of college students. Constructing the trinity system of prevention and intervention of college students' psychological crisis is not only the professional and normative requirement of college to prevent and intervene in students' psychological crisis, but also the realistic conditions for the establishment and implementation of it. It is meaning for the prevention and intervention of college students' psychological crisis and college students' mental health education.

\section{REFERENCES}

[1] Zang Weiwei, Qin Tao. Research on Psychological Crisis Strategy of College Counselors [J]. Forum on Education and Teaching, 2015,(03).

[2] Su Binyuan, Zhang Jieting. Identification of College Students' Psychological and Behavioral Problems: Based on Latent Profile Analysis [J]. Psychological Development and Education,2015,(03).

[3] Zang Weiwei, Qin Tao. Research on Psychological Crisis Strategy of College Counselors [J]. Educational Observation,2015,(02).

[4] Liu Haibo, Research on the Influencing Factors and Training Strategies of College Students' Mental Health[J]. The Front,2014,(01).

[5] Wang Qun, Lin Lei. Research on the prevention and intervention system of College Students' Psychological Crisis [J]. Management Science, 2013,(02).

[6] Song Ge. On the Prevention of College Students' Psychological Crisis[J]. Educational Exploration, 2012,(02).

[7] Wang Yinxiong. A review of Factors Affecting College Students' Mental Health in the New Era [J]. Journal of Shanxi Finance and Economics University, 2011,(05).
[8] Wang Huilin. Reflections on College Counselors' Participation in Psychological Crisis Intervention [J]. Ideological Education Research, 2009,(06).

[9] Ren Xinhong Influencing Factors and Corresponding Measures of College Students' Mental Health [J]. Ideological and Political Work, 2009,(05). 